\title{
Integrated Optics
}

\author{
Giancarlo C. Righini \\ Nello Carrara Institute of Applied Physics \\ Via Madonna del Piano 10 \\ 50019 Sesto Fiorentino, Firenze, Italy \\ E-mail: giancarlo.righini@cnr.it
}

The first demonstration of the laser in May $1960^{1}$ opened the way to the development of lightwave technology. Almost 20 years later, the production of very-low-loss optical fibers ${ }^{2}$ made guided-wave optical communication systems a reality. One of the problems associated with the development of longhaul systems was obviously related to the introduction in the transmission line of a number of repeaters, able to recondition and to reamplify the optical signal. The solution offered by conventional optics was unsatisfactory, due to the size and electrical power consumption, as well as to the critical effects of temperature variations, mechanical vibrations, and the presence of moisture. Similar problems existed for signal distribution to end users, which required a very large number of splitters and switches. The alternative first suggested by S. E. Miller, a researcher at Bell Laboratories, was to miniaturize the repeater, integrate all the components onto a single chip, and interconnect them via optical waveguides: the concept of integrated optics was born. ${ }^{3}$

Technology of materials and devices has progressed a great deal since then, and terminology has also slightly changed: it is now common to speak about integrated optoelectronics or integrated photonics rather than integrated optics. The basic aim, however, has not changed with time: to integrate as many optical and electronic functions as possible into a chip as small as possible. One of the most complex photonic integrated circuits (PICs) reported to date is an $\ln P$ monolithic tunable optical router with 8 input and 8 output ports, capable of $40 \mathrm{~Gb} / \mathrm{s}$ operation per port. ${ }^{4}$ The size of this $\mathrm{PIC}$ is $4.25 \times 14.5 \mathrm{~mm}$, and it includes more than 200 functional elements integrated on-chip. In recent years, besides the telecommunications applications, a continuously growing interest has been focused on the field of integrated optical sensors, especially for biomedical sensing. Hot topics in this area concern, for instance: (i) the integration of surface plasmon resonance (SPR) sensors in a waveguide configuration (waveguide-based SPR sensors can accommodate several sensing elements on a single platform, thus allowing us the development of highly integrated, multichannel, and robust sensing devices); see, for instance, Ref. 5; (ii) the integration of high-Q microresonators with optofluidics for ultrasensitive spectroscopic measurements (which could enable miniaturized, low-cost, fully automated, and massively parallel devices for lab-on-a-chip applications); see, for instance, Ref. 6; and (iii) the combination of ultrahigh- $Q$ whispering gallery mode microresonators with optical waveguides for the implementation of very sensitive biosensors; see, for instance, Ref. 7.

Several books ${ }^{8-13}$ and a huge number of papers on this subject have been published in the 40 years since the sem- inal paper by P. K. Tien in 1971, which described not only the physics of guided waves in thin films and the prismcoupling method, but also some very early nonlinear optical phenomena in integrated optics. ${ }^{14}$ An early 2011 search of the SPIE Digital Library on the term "integrated optics" within Abstract/Title/Keyword produced about 2000 papers; of those, more than $10 \%$ were published in Optical Engineering. A similar search on the ISI Web of Knowledge produced 3810 journal articles and 3655 proceedings papers. The histogram in Fig. 1 shows the publication rate in the last 20 years; it appears evident that the number of publications on integrated optics stayed high and almost constant, on the order of 500 per year, in the last 7 to 8 years, thus testifying to the continuous importance of this R\&D field.

This Optical Engineering special section aims at providing just a flavor of topics of contemporary interest in this area, covering a variety of applications and concepts. Glass still is one of the main materials for integrated optical circuits, and several papers in this special section are concerned with it. lon exchange is likely the most common fabrication technique of optical waveguides in glass substrates; the paper by Tervonen et al. presents a comprehensive review of this process, while Hassanzadeh and Mittler discuss the optimization of a two-step ion exchange $\left(\mathrm{K}^{+}-\mathrm{Na}^{+}\right.$followed by an $\mathrm{Ag}^{+}$ $\mathrm{Na}^{+}$) for producing low-loss waveguides with high surface refractive indices. In some glasses, however, ion exchange is not effective, and other waveguide fabrication techniques

\section{Published Items in Each Year}

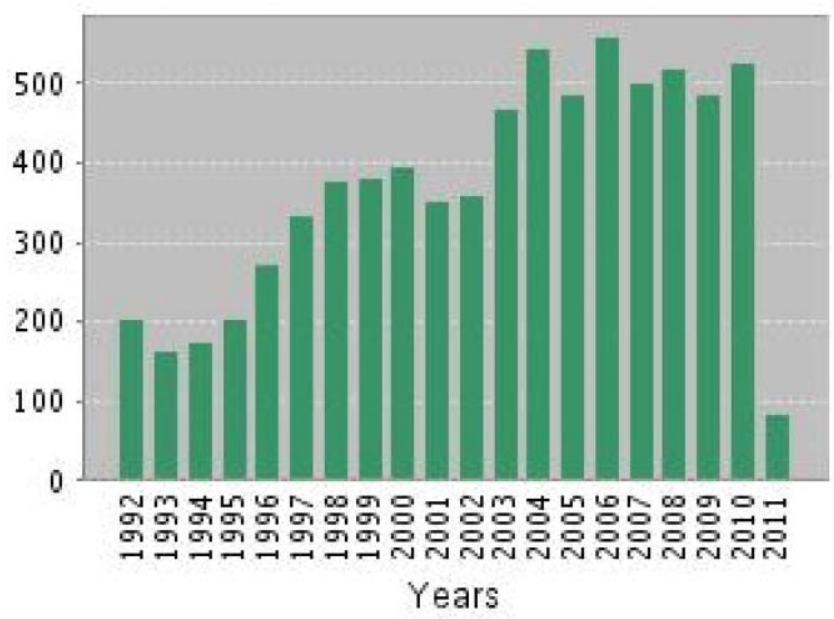

Fig. 1 Number of publications on integrated optics in the last 20 years (from a search on ISI Web of Science). 
have to be used: the paper by Berneschi et al., for instance, presents an approach based on nitrogen ion implantation at $1.5 \mathrm{MeV}$ beam energy to produce waveguides in tellurite glasses, which have gained widespread attention because of their potential as hosts of rare-earth elements for the development of fiber and integrated-optic amplifiers and lasers covering all the main telecommunication bands. Another aspect of rare-earth-doped glasses is discussed in the paper by Chiasera et al., who studied the luminescence properties of $\mathrm{Er}^{3+}$ in glass-ceramic waveguides, confirming the advantages of this class of nanostructured systems.

Among the many integrated optical components, Bragg grating structures definitely play important functions; integrated Bragg reflectors based on liquid-crystal waveguides that can be electro-optically tuned over a large wavelength spectral range are described in the paper of Asquini et al. Another important class of devices is constituted by modulators and optical switches: Wen et al. investigate the issues of velocity and impedance matching for electro-optic modulators in polymeric materials. Thermo-optic switches are reviewed by Coppola et al., while Flores-Rosas et al. present experimental results on optical switches based on stimulated Raman scattering, and Tao et al. discuss thermal stability of a nonlinear optical switch based on an asymmetric three-core nonlinear directional coupler with a variable Gaussian coupling coefficient.

Finally, two examples of integration are offered by the paper of Van der Tol et al., who designed a Brillouin optical time domain reflection read-out unit with low-cost potential, to be fabricated in an InP/InGaAsP/InP double heterostructure, and the paper by Wiederkehr et al., who developed a broadband spectroscopic platform based on single-mode integrated optical waveguides for the study of molecular surface adsorption processes.

In spite of the fact that this special section can offer only a very limited view of the ongoing research and development efforts in this vast field, I hope that the papers presented here can be a useful reference for both experts and newcomers in the active and still growing area of integrated optics and photonics. Sincere thanks are due to the peer reviewers, the SPIE staff who managed submissions and publication processes, and the editor of Optical Engineering, Ron Driggers, whose suggestion led to this special section.

\section{References}

1. T. H. Maiman, "Stimulated optical radiation in ruby," Nature 187, 493 (1960).

2. T. Miya, Y. Terunuma, T. Hosaka, and T. Miyashita, "Ultimate low-loss single-mode fibre at $1.55 \mu \mathrm{m}$," Electron. Lett. 15, 106 (1979).

3. S. E. Miller, "Integrated optics: an introduction," Bell Syst. Tech. J. 48 2059 (1969)

4. S. C. Nicholes, M. L. Masanovic, B. Jevremovic, E. Lively, L. A. Coldren, and D. J. Blumenthal, "An $8 \times 8 \mathrm{InP}$ monolithic tunable optical router (MOTOR) packet forwarding chip," J. Lightwave Tech. 28, 641 (2010).

5. E. K. Akowuah, T. Gorman, S. Haxha, and J. V. Oliver, "Dual channel planar waveguide surface plasmon resonance biosensor for an aqueous environment," Optic. Express 18, 24412-24422 (2010).

6. A. Nitkowski, A. Baeumner, and M. Lipson, "On-chip spectrophotometry for bioanalysis using microring resonators," Biomed. Optic. Express 2, 271-277 (2011).

7. S. Soria, S. Berneschi, M. Brenci, F. Cosi, G. Nunzi Conti, S. Pelli, and G. C. Righini, "Optical microspherical resonators for biomedical sensing," Sensors 11, 785-805 (2011).

8. T. Tamir, Ed., Integrated Optics (Springer-Verlag, Berlin, 1975).

9. R. G. Hunsperger, Integrated Optics: Theory and Technology, (SpringerVerlag, Berlin, 1982)

10. H. Nishihara, M. Haruna, and T. Suhara, Optical Integrated Circuits, (McGraw-Hill, New York, 1989).

11. M. A. Mentzer, Principles of Optical Circuit Engineering (Marcel Dekker, New York, 1990)

12. S. I. Najafi, Ed., Introduction to Glass Integrated Optics (Artech House, Norwood, MA, 1992).

13. K. K. Wong and M. Razeghi, Integrated Optics and Optoelectronics (SPIE Press, Bellingham, WA, 1993)

14. P. K. Tien, "Light waves in thin films and integrated optics," Appl. Opt. 10, 2395-2413 (1971).

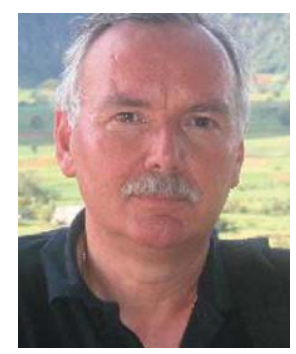

Giancarlo C. Righini was on the staff of the National Research Council of Italy (CNR) at Nello Carrara Institute of Applied Physics (IFAC) in Florence, from 1972 to 2010 . He retired from CNR in September 2010 and is now an associate scientist at IFAC. He was research director at IFAC from 1991 to 2010; director of CNR-GNEQP from 1983 to 1996; and director of CNR National Department of Materials and Devices from 2006 to 2009. Since 2004 he has been a contract professor of optoelectronics at Florence University. His activity has been largely devoted to glass integrated optics, but extended to holography, optical signal processing, and fiber optics. He has published over 400 papers and coauthored 6 patents. He is a Fellow of SIOF, EOS, OSA, and SPIE. 